\title{
Hybrid mapping for the assistance of teleoperated grasping tasks
}

\author{
Luca Colasanto, Raúl Suárez, Member, IEEE, and Jan Rosell, Member, IEEE
}

\begin{abstract}
Teleoperating a robotic hand with the aid of a sensorized glove presents some particular problems. One such problem is due to the kinematic differences between the human hand and the robotic hand, which do not allow a simple direct mapping of the sensor readings from the glove to the robotic hand. This problem is addressed with different types of mapping, but none of them is of general use. This paper proposes two new mappings within two existing mapping types, as well as a new hybrid mapping that combines the best features of these existing mapping types. This hybrid mapping allows intuitive free space movements (where the gesture is more important than the precise positions of the fingers) and grasp movements (where the precise positions of the fingers is more important than the gesture), despite kinematic differences between the human hand and the robotic hand. The approach has been implemented and some illustrative examples are presented in the paper.
\end{abstract}

Index Terms-Teleoperation, Grasping, Robotic Hands, Sensorized Gloves, Fuzzy Systems.

\section{INTRODUCTION}

$\mathbf{T}$ ELEOPERATION of complex devices has advanced significantly in the last years, both from the point of view of technological developments that allows communications and exchange of information with higher velocity, as well as from the point of view of algorithm developments to process such information and properly control the system. A detailed review of the state of the art in teleoperation together with a description of the involved problems and the main current applications can be found in [1]. Nevertheless, despite these advances, some applications have particular problems that still need more practical solutions, such as in the case of teleoperation of anthropomorphic robotic hands.

Teleoperation of an anthropomorphic robotic hand-arm system requires the coordinated movement of all hand and arm joints to perform a given task. Talking only about the hand, this implies commanding a number of degrees of freedom (dof) usually ranging from 12 (case of hands with 4 fingers with 3 independent dof each one) to 25 (case of hands with 5 fingers with 4 independent dof each one plus one additional dof in the palm). This means that it is very hard, if not impossible, for a human being to generate the setpoint of each joint working in such a high dimensional joint space. Thus the more intuitive way to do it is by executing the desired movements with the human hand, capturing this information in some way, and processing it to command the robotic hand to emulate the

R. Suárez and J. Rosell are with the Institut d'Organització i Control de Sistemes Industrial (IOC) at the Universitat Politècnica de Catalunya (UPC), Barcelona, Spain. Luca Colasanto was with the IOC-UPC during the development of the work. (raul.suarez@upc.edu)

This work was partially supported by the Spanish Government through the projects DPI2010-15446 and DPI2011-22471. operator movements. Nevertheless, two particular problems of this approach are:

- identification of the configurations of the human hand,

- proper imitation of these configurations with the robotic hand.

The first of these problems has been mainly addressed using a vision system or a sensorized glove to identify the human hand configurations. Vision systems are used in applications were the gesture of the hand transmits relevant information, as for instance in hand gesture/sign languages, computer interfaces and computer games. Representative works in this line are applied to identify orders for an interactive system, using motion patterns and Petri nets [2], stereo vision [3], or analyzing the contour of the hand [4]. Hand pose recognition using vision was also applied to command an industrial robot arm [5] as well as to command a mechanical hand [6]. Nevertheless, the use of vision in most of the robotic applications requires the addition of special marks on the hand to facilitate the pose identification, and has the disadvantage that visual occlusions are quite frequent during the hand movements. A sensorized glove was also used to identify hand signals, for instance using neural nets and fuzzy rules [7] and a graph matching approach [8], to identify hand poses using Principal Component Analysis and discriminant functions [9], to teleoperate robotic anthropomorphic [10] and non-anthropomorphic [11] hands, and to extract information about how the humans perform grasping actions [12] [13]. The problem was also addressed in the scope of programming by demonstration, for instance using neural nets [14] or a nearest neighbor algorithm and some patterns defined by a training session [15]. Another interesting approach to identify hand postures is based on the measurements of electromyogram signals (EMG) from the forearm [16] [17], with the main application of commanding prosthetic hands.

The second problem does not have yet a general solution. Even when the anthropomorphic robotic hand has the same structure of the human hand, an exact copy of the joint angles of the human hand will result in different relative positions of the fingertips due to the kinematic differences between them. This means that it is highly probable that, if the human operator performs a precision grasp of an object, the robotic hand would fail to reproduce it in this way. This problem can be partially reduced by providing the operator with some visual and/or haptic feedback of the teleoperated environment, so that he/she can properly modify his/her hand configuration to properly perform the task. This also requires a relatively extensive training of the operator. In order to map the information obtained from the human hand to the mechanical 
hand mounted on the robot arm, the following three mapping methods were presented in the literature (pionering works [18] [19] and more recently [20]):

- Joint-to-joint mapping. Each joint of the glove is directly associated with a joint of the mechanical hand. Advantage: simplicity. Disadvantage: problems due to differences in the relative positions of the fingers in the human hand with respect to the mechanical hand, due largely to the differences in the kinematic parameters. E.g.: [13], where this mapping is used to obtain a simplified model of the joint space (13-dimensional) of a 4-fingered robotic hand for hand motion planning applications.

- Pose mapping. A particular pose of the hand is associated with a predefined pose of the mechanical hand. Advantage: the pose of the mechanical hand can be preprogrammed. Disadvantage: the pose of the human hand must be properly identified, and the equivalent pose of the mechanical hand must be properly choosen. E.g.: [21], where a set of 23 poses of the human and robotic hands are associated, and from which a linear transformation is obtained to map other poses (the size of the set of associate poses is constrained by the number of joints of the robotic hand and the number of measured joints in the operator's hand).

- Point-to-point mapping. The position of a particular point in each fingertip is replicated by a predefined point in the corresponding fingertip of the mechanical hand. Advantage: the relative positions of the mechanical hand fingertips are more precisely defined. Disadvantage: it is necessary to solve the inverse kinematics of the mechanical hand, which may be a time-consuming operation. E.g.: [10], where the direct kinematics is used to determine a point of the operator fingertips based on the glove information and then the inverse kinematics is used to obtain the corresponding configuration of an anthropomorphic robotic hand.

Dealing with these problems, this work proposes an approach that uses the information obtained from a sensorized glove to command a four-finger robotic hand. The experimental hardware is detailed in Section II.

The proposed approach merges the first two types of mapping to obtain an hybrid mapping that can be used to command hand movements in the free space as well as for an assisted guidance to perform a grasp in an intuitive way. This is valid for power grasps, where stability and security are predominant with little or null ability to impart motions with the fingers (they are distinguished by large areas of contact between the grasped object and the fingers and palm), as well as for precision grasps, where sensitivity and dexterity are predominant (in general the object is held with the tips of the fingers and thumb) [22], [23].

The new joint-to-joint mapping developed takes into account the differences between the human hand and the mechanical hand to try to minimize the disadvantage of this type of mapping while preserving the mapping simplicity. As it is not exactly a one-to-one mappings we refer to it as jointspace mapping. It is able to reproduce free hand movements

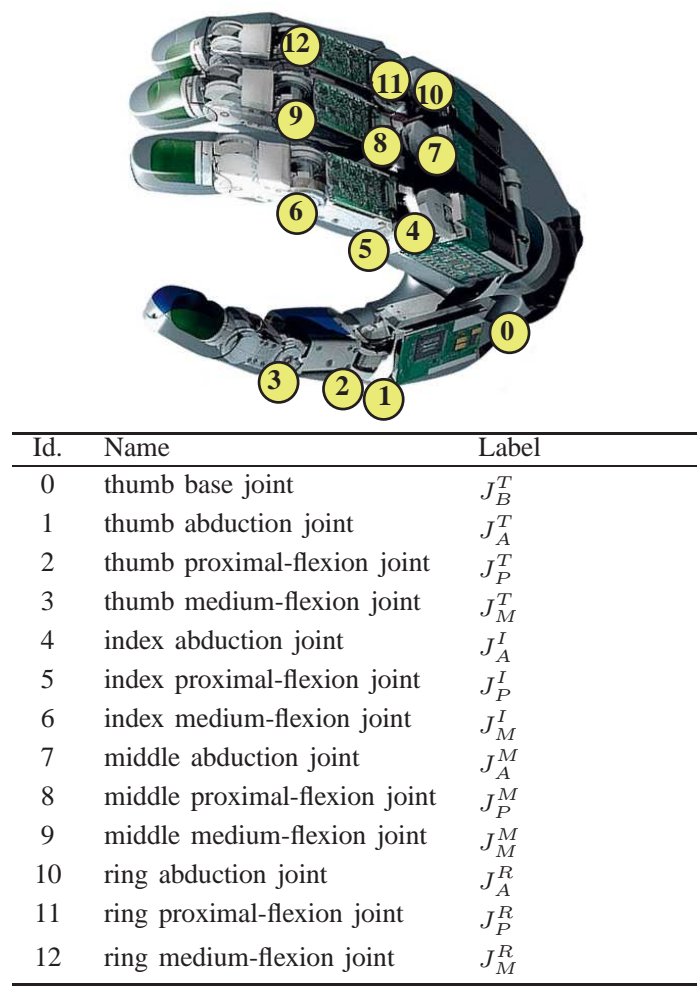

Fig. 1. The anthropomorphic mechanical hand SAH and joint labels, the supraindices indicate the corresponding fingers ( $T$ :Thumb, I=Index, $\mathrm{M}=$ Middle and $\mathrm{R}=\mathrm{Ring}$ ) and subindices the corresponding joints ( $\mathrm{B}=\mathrm{Base}$, $\mathrm{A}=$ Abduction, $\mathrm{P}=$ Proximal and $\mathrm{M}=$ Medium).

and power grasps but it is still unprecise and difficult to use for precision grasps. This mapping is described in detail in Section III.

The new pose mapping developed uses fuzzy logic [24] to identify some poses of the human hand, and associates them with predefined poses of the mechanical hand, allowing precision grasps to be performed according to the human operator movements. We refer to it as fuzzy-based pose mapping, and it is described in detail in Section IV.

The joint-space mapping and the fuzzy-based pose mapping are then merged into a single system, which we call hybrid mapping. In this system, joint space mapping is continuously executed until the fuzzy system identifies a particular grasping pose of the human hand and the corresponding predefined pose is executed in the mechanical hand. This mapping is described in Section V.

In Section VI, some examples are given to illustrate the results of approach, and finally, in Section VII, some conclusions and future work are presented.

\section{EXPERIMENTAL SET-UP}

The experimental set-up used in this work involves: a) an anthropomorphic mechanical hand, b) an industrial robot, c) a sensorized glove, d) a wrist tracker, e) a hand/robot simulator connected with the real elements. The main relevant details about these elements are:

a) Anthropomorphic mechanical hand. We use the Schunk Anthropomorphic Hand (SAH) [25], shown in Fig. 1, 


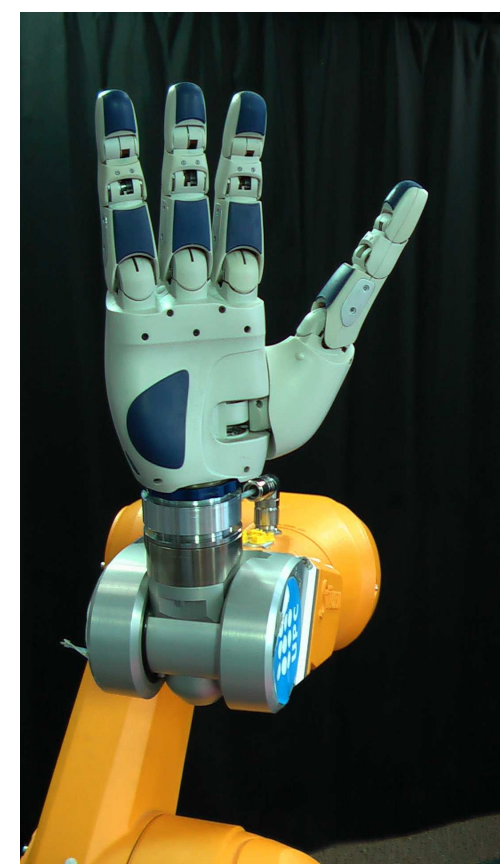

Fig. 2. Industrial robot Stäubli TX 90 with the mechanical hand SAH.

which is based on the DLR hand [26]. It has four identical fingers with four joints each one (called abduction, proximal flexion, medium flexion and distal flexion), and one of them is prepared to act as the opposing thumb and it is equipped with an additional joint (called thumbbase joint), that moves the whole thumb with respect to the palm. In all the fingers the distal and medium flexion joints are mechanically coupled, thus there are a total of 17 joints with only 13 independent $d o f$.

b) Industrial robot. The hand is assembled on an industrial robot Stäubli TX-90, as shown in Fig. 2. It is a general purpose six dof robot arm.

c) Sensorized glove. We use a sensorized glove CyberGlove (shown in Fig. 3). It is a fully instrumented glove that provides 22 joint-angle measurements using resistive bendsensing technology, including three flexion sensors per finger, four abduction sensors between the fingers, a palm-arc sensor, and two sensors to measure the flexion and the abduction of the wrist.

d) Tracker. The magnetic wrist tracker with six dof Flock of Birds from Ascension Technology Corporation is used to capture the position and orientation of the user wrist with respect to a global reference frame, allowing a mapping of the displacements of the user arm to the robot arm (the description of this mapping is outside the scope of this work).

e) Hand and robot simulator. A simulation tool has been programmed in our lab that allows: planning and simulation of collision-free paths of the hand-arm system [27](Fig. 4); control and calibration of input devices (glove and tracker); commanding of output devices (the robot and the mechanical hand); on-line graphical visualization of the mechanical hand movements associated

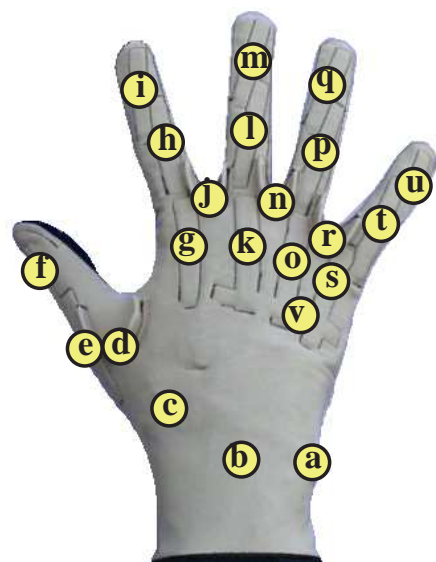

\begin{tabular}{cll}
\hline Id. & Name & label \\
\hline a & palm arc sensor & unused \\
b & Palm sensor & unused \\
c & thumb base sensor & $S_{B}^{T}$ \\
d & thumb-index abduction sensor & $S_{A}^{T I}$ \\
e & thumb medium-flexion sensor & $S_{M}^{T}$ \\
f & thumb distal-flexion sensor & $S_{D}^{T}$ \\
g & index proximal-flexion sensor & $S_{P}^{I}$ \\
h & index medium-flexion sensor & $S_{M}^{I}$ \\
i & index distal-flexion sensor & $S_{D}^{I}$ \\
j & index-middle abduction sensor & $S_{A}^{I M}$ \\
k & middle proximal-flexion sensor & $S_{P}^{M}$ \\
l & middle medium-flexion sensor & $S_{M}^{M}$ \\
m & middle distal-flexion sensor & $S_{D}^{M}$ \\
n & middle-ring abduction sensor & $S_{A}^{M R}$ \\
o & ring proximal-flexion sensor & $S_{P}^{R}$ \\
p & ring medium-flexion sensor & $S_{M}^{R}$ \\
q & ring distal-flexion sensor & $S_{D}^{R}$ \\
r & middle-little abduction sensor & unused \\
s & little proximal-flexion sensor & unused \\
t & little medium-flexion sensor & unused \\
u & little distal-flexion sensor & unused \\
\hline & &
\end{tabular}

Fig. 3. Sensorized glove used to capture the operator hand workspace and sensor labels, the supraindices indicate the corresponding fingers (T:Thumb, $\mathrm{I}=$ Index, $\mathrm{M}=$ Middle, $\mathrm{R}=$ Ring and $\mathrm{L}=\mathrm{Little})$ and subindices the corresponding sensorized joints ( $\mathrm{B}=\mathrm{B}$ ase, $\mathrm{A}=\mathrm{Abduction}, \mathrm{P}=$ Proximal and $\mathrm{M}=\mathrm{Medium}$ ).

with the human operator hand movements captured with the sensorized glove (Fig.5); and on-line visualization of the robot movements associated to the movements of the human operator wrist, which are captured using the tracker.

\section{JOINT SPACE MAPPING}

The joint space mapping introduced in this work is an enhanced version of the simple joint-to-joint mapping approach, designed to minimize the differences in the relative positions of the fingertips of the human hand and those of the mechanical hand due to the differences in the kinematic structure and parameters. The main adjustments introduced are due to the following reasons:

- The kinematic parameters and joint range of motion of the human hand and the mechanical hand are different.

- The kinematic structures differ largely in the palm, which is rigid in the $\mathrm{SAH}$ hand, and flexible in the human 


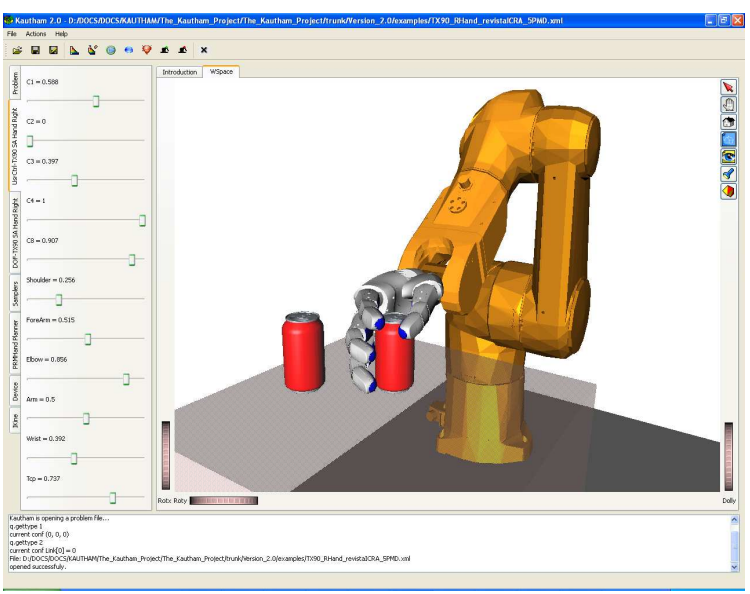

Fig. 4. Hand and robot simulator including the planning environment.

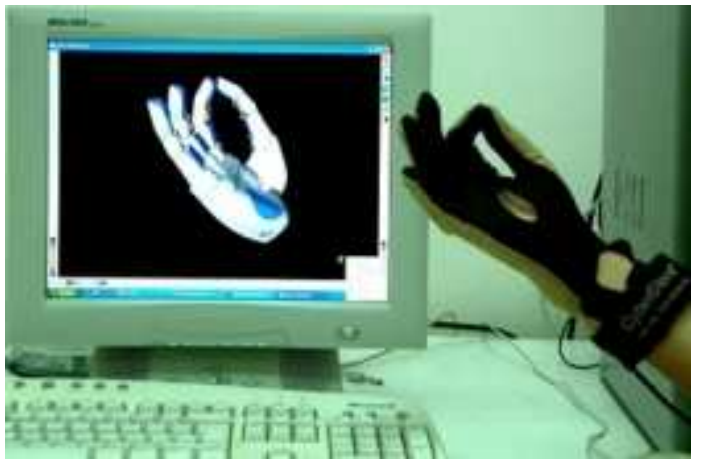

Fig. 5. Human hand with the sensorized glove connected to the mechanical hand simulator used in the data acquisition procedure.

hand. This structural difference mainly affects thumb movements.

- The sensors of the glove cannot be clearly mapped to joints in the mechanical hand (there are 22 sensors in the glove and only 13 independent joints in the mechanical hand). This discrepancy is particularly important in the abduction joints, and in the distal and middle flexion joints that are coupled in the SAH hand.

The following subsections explain how these problems are treated for the different finger joints, in order to minimize their effect and obtain a better mapping. The nomenclature used for the joints of the mechanical hand and for the sensors of the glove is presented in Fig. 1 and 3, respectively.

\section{A. Joint limits}

All dataglove sensor values have a range between 0 and 255 . These values are conservative and cover the real motion of the human hand (i.e. the real ranges obtained when the user wears the glove are always smaller). For this reason, the minimum and maximum actual values $\left(x_{\min }\right.$ and $\left.x_{\max }\right)$ are obtained experimentally by capturing the values obtained by different users performing motions covering the whole hand workspace. The experimental range of the dataglove sensors are used to obtain normalized values from real data:

$$
\operatorname{norm}(x)=\operatorname{Sat}\left(\frac{x-x_{\min }}{x_{\max }-x_{\min }}\right)
$$

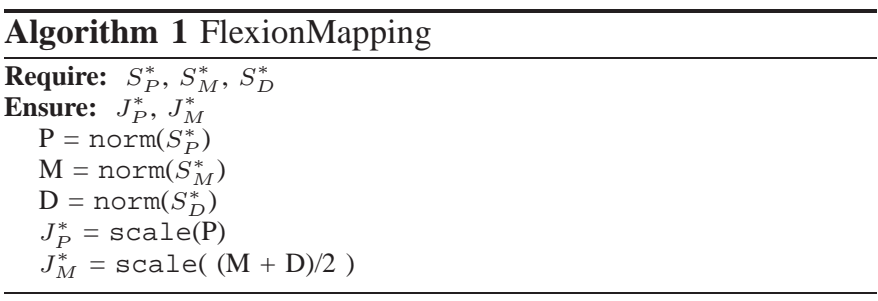

where $\operatorname{Sat}(x)$ is a saturation function such that $\operatorname{Sat}(x)=0$ if $x<0, \operatorname{Sat}(x)=1$ if $x>1$ and $\operatorname{Sat}(x)=x$ otherwise. This normalization permits better use of glove sensors measurement ranges, with the saturation function limiting those few cases in which the actual sensed value lies outside the range $\left[x_{\min }, x_{\max }\right]$.

The SAH hand has divergences between the minimum and maximum joint values provided by the manufacturer and the actual values obtained experimentally $\left(y_{\min }\right.$ and $\left.y_{\max }\right)$, that result in a smaller range. Therefore the measured values are the limits used to obtain scaled values from normalized data:

$$
\operatorname{scale}(y)=y_{\min }+y\left(y_{\max }-y_{\min }\right) \quad 0 \leq y \leq 1
$$

The functions norm and scale are used in the mapping algorithms detailed in the following subsections.

\section{B. Flexion joints}

The distal and medium joints of the SAH hand are mechanically coupled. This fact does not imply a loss of hand anthropomorphism, as these phalangeal joints are also often coupled in the human hand. Many people are unable to move the distal joint independently from the medium joint in absence of constraints.

Algorithm 1 takes this into account when mapping the flexion joint values from the glove to the joints of the $\mathrm{SAH}$ hand. The character "*" used in the variables of the Algorithm stands for any of the letters I, M or R that refer, respectively, to the index, the middle and the ring fingers. The thumb is treated separately in Section III-D.

\section{Abduction joints}

The dataglove does not give an absolute abduction measurement, but merely the angle between two consecutive fingers. This is considered in Algorithm 2, based on experimental observation of the human hand when performing abduction movements and corresponding sensor readings. Its main features are the weighted distribution of the sensor values between the abduction joints (illustrated in Fig. 6), and the saturation introduced to avoid collisions. This saturation is implemented using a threshold $\mu$ that limits the amount that the index and ring fingers can move depending on the position of the middle finger.

\section{Thumb finger}

The thumb and the other fingers of the SAH hand are identical, which is not the case in the human hand. For this reason, the thumb must be treated in a different way, as has been done in the mappings proposed for the thumb detailed 


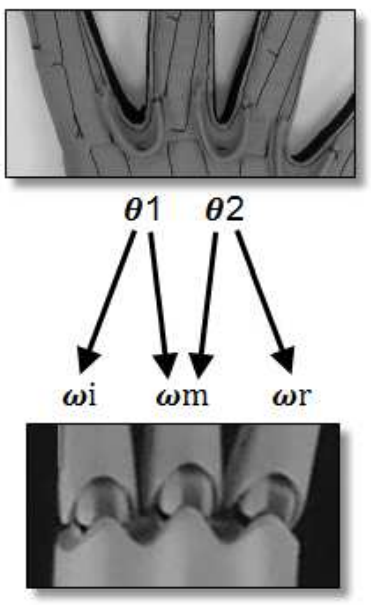

Fig. 6. Abduction mapping $\left(\omega_{i}=\theta_{1}, \omega_{m}=0.5+\left(\theta_{2}-\theta_{1}\right) / 2\right.$ and $\left.\omega_{r}=1-\theta_{2}\right)$.

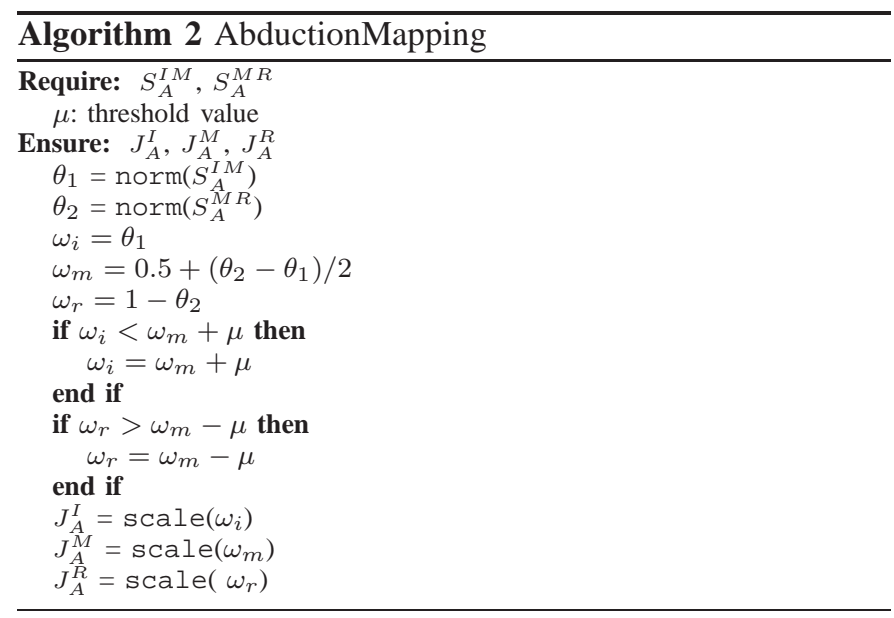

in Algorithms 3 and 4. In these mappings the data from the abduction sensor between the thumb and the index $S_{A}^{T I}$ are used for the thumb proximal-flexion joint $J_{P}^{T}$ of the SAH hand, the data from the distal and medium flexion sensors $S_{D}^{T}$ and $S_{M}^{T}$ are used for the thumb medium-flexion joint $J_{M}^{T}$, and the thumb base sensor $S_{B}^{T}$ is used for both the abduction and base joints $J_{A}^{T}$ and $J_{B}^{T}$.

The thumb base movement of the $\mathrm{SAH}$ hand is very different from the movement of the human hand. This is tackled in Algorithm 4 where the abduction joint of the thumb and the thumb-base joint are set in a coupled way. In this algorithm the function minLimitJointValue $\left(J_{B}^{T}\right)$ returns the minimum value of the range of $J_{B}^{T}$.

\section{FUZZY-BASED POSE MAPPING}

The aim of this mapping is to ensure that a desired grasp movement of the mechanical SAH hand is executed when a particular type of grasp is executed by the operator. In this work we consider nine types of particular grasps, described below in Subsection IV-A.

Executing a precision grasp needs high precision finger coordination, including the determination of collision free paths. One way to ensure the observance of these features,
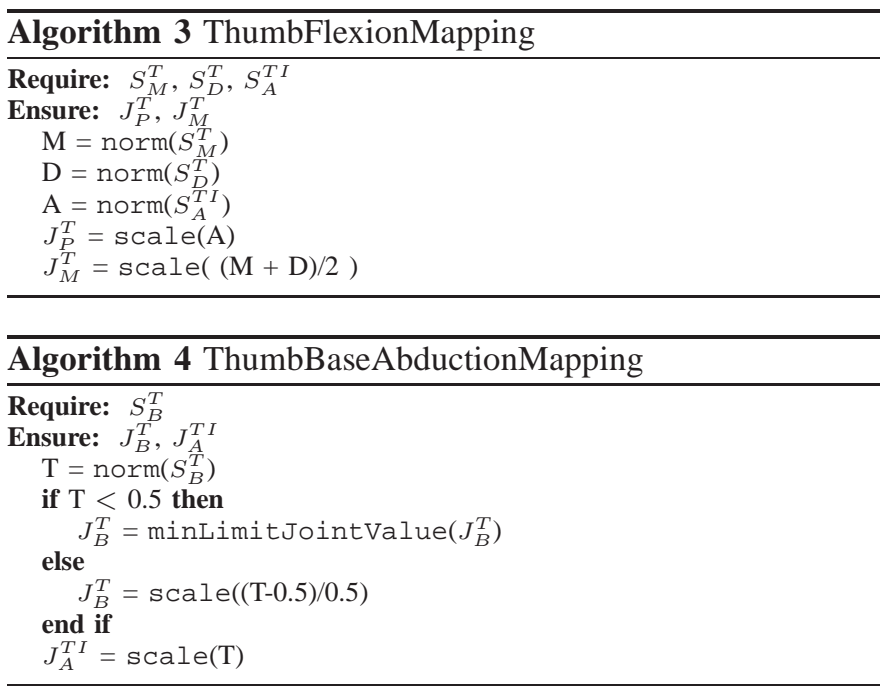

is to establish a predefined relation between the grasp being executed by the operator (measured with the sensorized glove) and the mechanical hand, i.e. a pose mapping, as described in paper introduction. One of the main difficulties in a pose mapping is related with the variability of the user hand poses, on one side, due to the differences between users, and on another due to the variance between different executions of the same task. Therefore, it is necessary to develop a system flexible enough to deal with this variability while ensuring a robust grasp identification. In this work we propose a new approach based on a fuzzy logic controller (FLC) [28]. A block schema of this mapping is shown in Fig. 7.

\section{A. Selection of the particular types of grasp}

In general, an object can be grasped using a large number of different hand poses. Selection of the appropriate pose depends on such factors as:

- The task to be performed (e.g. the grasp has to compensate large reaction forces or be able to produce fine object movements).

- The grasped object (e.g. the shape, the slipperiness, and the fragility of parts of the object constrain the regions where fingers should be placed).

- The hand kinematics and strength (e.g. the maximum grasp force and maximum opening of the fingers also impose conditions on the proper grasp).

Considering all these aspects together is not an easy task, and frequently the grasp problem is solved as a set of independent sequential problems, leading to far from optimal solutions.

The study of human grasping capabilities has been also an area of interest in other fields such as, for instance, hand

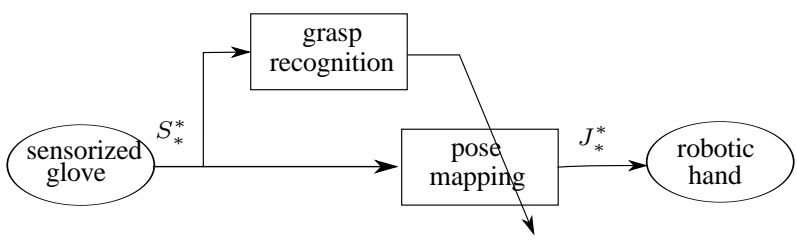

Fig. 7. General diagram of the fuzzy-based pose mapping. 


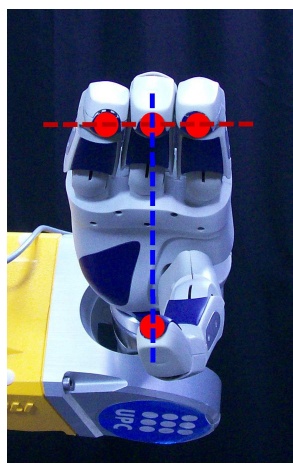

a)

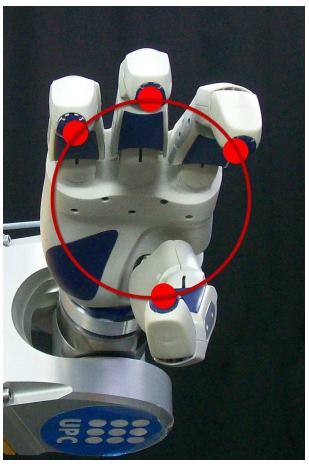

b)
Fig. 8. Examples of: a) prismatic grasp, b) circular grasp.

surgery, design of prosthetic devices, and quantification of disability in individuals with congenital defects or injuries. As a result, there is a substantial and empirical medical literature related to grasping, from which six types of grasp were identified [29]: cylindrical, fingertip, hook, palmar, spherical, and lateral. Additionally, another classification [22] suggests a scheme that divides grasps into two categories: power grasps and precision grasps (as they were defined in Section I).

Based on these classifications, Cutkosky [23] developed a hierarchical tree of grasps. The grasp-root is broken up into the two main branches: power and precision grasps. Precision grasps are divided into prismatic grasps and circular grasps, which are distinguished by the fingertip positions. In prismatic grasps, fingertips are aligned defining a segment and the thumb tip is moved along the bisector of this segment (Fig. 8a). In circular grasps the tips of the fingers and the thumb are all placed along a circumference (Fig. 8b).

Following the Cutkosky's taxonomy, the nine types of precision grasps covering the common grasp poses have been selected here as candidates for the pose mapping (i.e to be executed with preprogrammed movements). These grasps include:

- Six prismatic grasps distinguished by the set of involved fingers and whether they are flexed or extended (Fig. 9):

$G_{T I e}$ : grasp with the Thumb and Index finger, extended.

$G_{T I M e}$ : grasp with the Thumb, Index and Middle fingers, extended.

$G_{\text {TIMRe }}$ : grasp with the Thumb, Index, Middle and Ring fingers, extended.

$G_{T I f}: \quad$ grasp with the Thumb and Index finger, flexed.

$G_{\text {TIMf: }}$ grasp with the Thumb, Index and Middle fingers, flexed.

$G_{\text {TIMRf: }}$ grasp with the Thumb, Index, Middle and Ring fingers, flexed.

- Three circular grasp distinguished by relative positions of the fingertips (Fig. 10):

$G_{\text {tripod: }}$ grasp with the Thumb, Index and Middle finger.

$G_{d i s k}:$ grasp with the Thumb, Index, Middle and Ring fingers, extended.

$G_{\text {sphere }}$ : grasp with the Thumb, Index, Middle and Ring fingers, flexed.
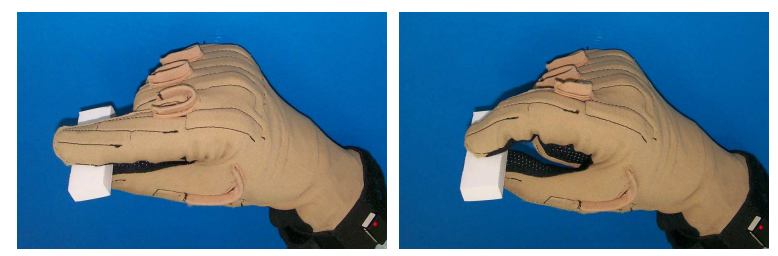

Extended $\left(G_{\text {TIe }}\right)$ and flexed $\left(G_{T I f}\right)$ Thumb-Index grasp
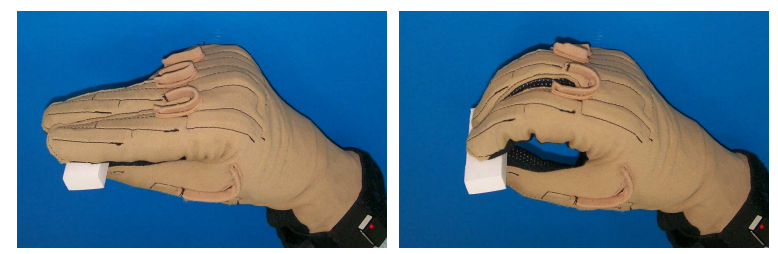

Extended $\left(G_{\text {TIMe }}\right)$ and flexed $\left(G_{\text {TIMf }}\right)$ Thumb-Index-Middle grasp
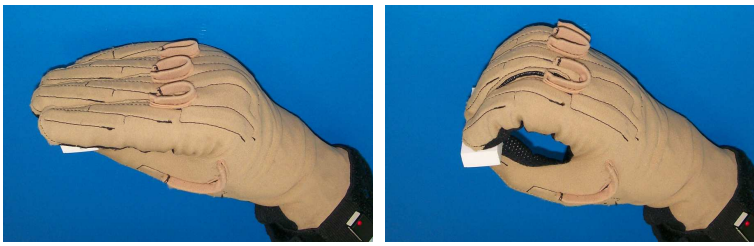

Extended $\left(G_{\text {TIMRe }}\right)$ and flexed $\left(G_{\text {TIMRf }}\right)$ Thumb-Index-Middle-Ring grasp

Fig. 9. Examples of the six types of considered prismatic grasps.

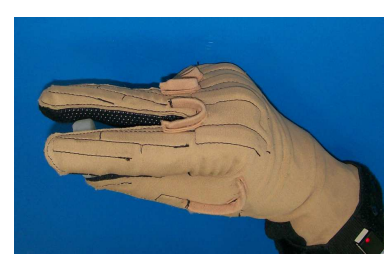

Tripod

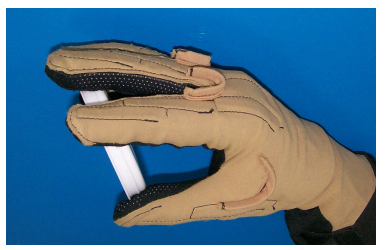

Disk

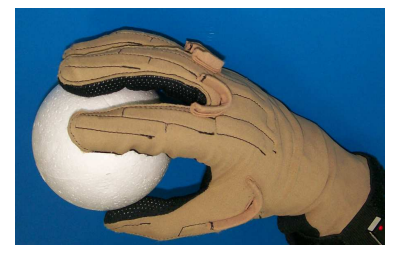

Sphere

Fig. 10. Examples of the three types of considered circular grasps.

\section{B. Recognition of the type of grasp}

The grasp recognition module receives the data from the sensorized glove, processes them with the FLC, and returns a code identifying the most likely configuration of the human operator.

The data from the sensorized glove is processed to obtain the following seven variables normalized between 0 and 1, that constitute the input to the FLC:

- $V_{R}^{T}$ : rotation of thumb across the palm;

- $V_{A}^{I M}$ : abduction angle between index and middle;

- $V_{A}^{M R}$ : abduction angle between middle and ring;

- $V_{F}^{T}$ : flexion of the thumb;

- $V_{F}^{I}$ : flexion of the index;

- $V_{F}^{M}$ : flexion of the middle;

- $V_{F}^{R}$ : flexion of the ring. 


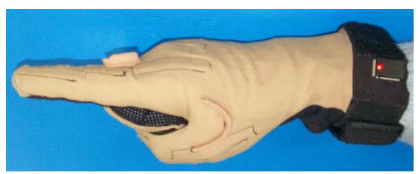

$V_{F}^{I}=0$

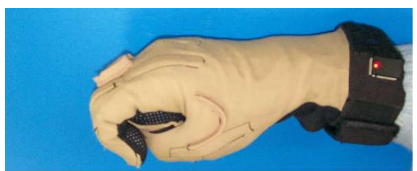

$V_{F}^{I}=0.85$

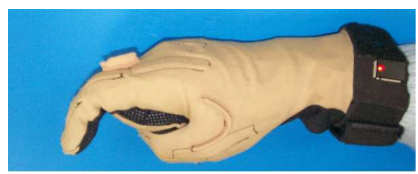

$V_{F}^{I}=0.50$

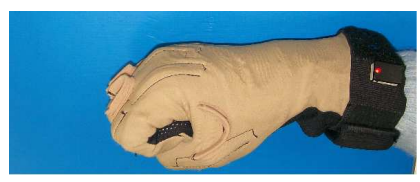

$V_{F}^{I}=1$
Fig. 11. Values of $V_{F}^{I}$ for different extensions of the index finger.

These variables are computed as,

$$
\begin{aligned}
V_{R}^{T}= & \operatorname{norm}\left(S_{B}^{T}\right) \\
V_{A}^{*}= & \operatorname{norm}\left(S_{A}^{*}\right) \\
V_{F}^{*}= & \operatorname{Sat}\left(a_{1} \operatorname{norm}\left(S_{D}^{*}\right)+a_{2} \operatorname{norm}\left(S_{M}^{*}\right)\right. \\
& \left.\quad+a_{3} e^{-20\left(1-\operatorname{norm}\left(S_{P}^{*}\right)\right)}\right)
\end{aligned}
$$

where $\operatorname{Sat}(x)$ is the saturation function introduced in Eq. 1 and $a_{1}, a_{2}$ and $a_{3}$ are non-negative weight constants.

$V_{R}^{T}$ and $V_{A}^{*}$ are straightforward, while $V_{F}^{*}$ was designed such that $V_{F}^{*} \simeq 0$ for the maximum extension of the finger and $V_{F}^{*}=1$ for the maximum flexion. $S_{D}^{*}$ and $S_{M}^{*}$ have different weights $a_{1}$ and $a_{2}$ in the computation of $V_{F}^{*}$ due to the different influence of the distal and middle joints on the total finger flexion (note that intuitively when the distal joint is completely flexed the finger is not considered significantly flexed, while when the middle joint is completely flexed the finger is intuitively considerably flexed). $a_{3}$ weights an exponential contribution of the proximal joint to the finger flexion, this is because it is necessary that this joint has a small contribution during most of its range and a rapidly growth for large flexion values. $a_{3}$ is set to zero for the thumb, because it has only 2 joints and the exponential contribution is not necessary. $a_{1}, a_{2}$ and $a_{3}$ are empirically fixed taking into account this reasoning (the used values are given in Section VI).

The value of $V_{F}^{*}$ is quite relevant for the fuzzy controller since it changes significantly between different types of grasps and changes just a little for hand configurations of the same type of grasp. Fig. 11 illustrates the variation of $V_{F}^{I}$ for different extensions of the index finger.

The seven variables $V_{*}^{*}$ are the inputs to the FLC, which determines the degree of belonging of each hand pose to each of the appropriate fuzzy sets.

All the variables $V_{*}^{*}$ except the thumb-roll $V_{R}^{T}$ are fuzzified with three membership functions, namely $\mathrm{A}, \mathrm{B}$ and $\mathrm{C}$, such that (see Fig. 12a):

A: include the lowest values;

B: include middle values;

$\mathrm{C}$ : include the highest values.

Regarding the variable $V_{R}^{T}$, it is fuzzified with only two membership functions, $\mathrm{A}$ and $\mathrm{B}$ for low and high values respectively (see Fig. 12b). The defuzzification is performed with the output membership function shown in Fig. 13.

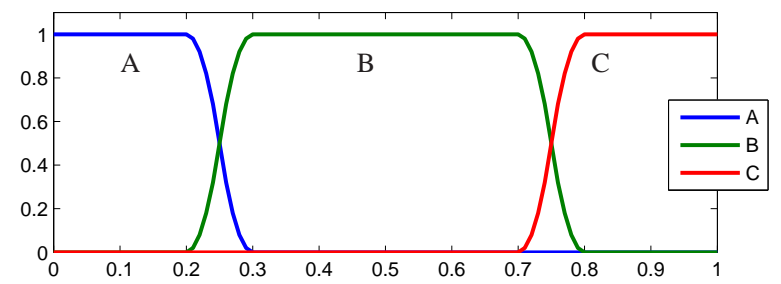

a)

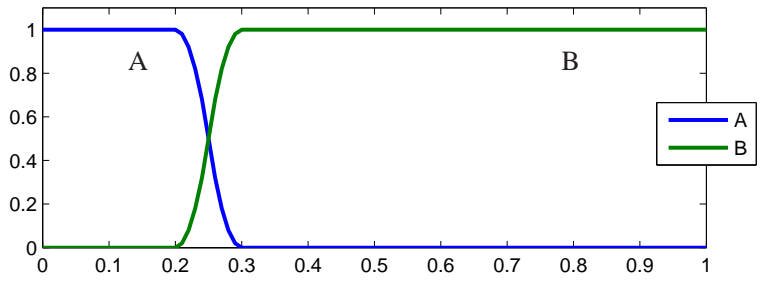

b)

Fig. 12. Input membership functions: a) for the general case $V_{*}^{*} \neq V_{R}^{T}$; b) for $V_{R}^{T}$.

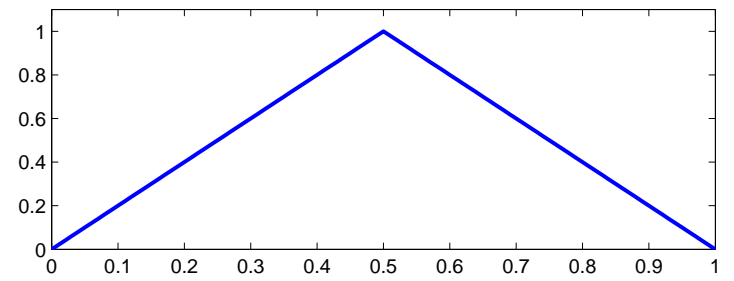

Fig. 13. Output membership function.

The used FLC implements a complete fuzzy interface system [30], using 12 fuzzy rules with the same structure:

$$
\begin{aligned}
& \operatorname{IF}\left(V_{1}=X_{1}\right) \text { and }\left(V_{2}=X_{2}\right) \text { and } \ldots \text { and }\left(V_{i}=X_{i}\right) \\
& \operatorname{THEN} \operatorname{return}\left(G_{*}\right)
\end{aligned}
$$

where:

$V_{i} \quad$ is an input variable to the FLC, i.e. $V_{i}=V_{*}^{*}$.

$X_{i} \quad$ is one of the corresponding membership functions (i.e. $X_{i} \in\{\mathrm{A}, \mathrm{B}, \mathrm{C}\}$ for the general case $V_{*}^{*} \neq V_{R}^{T}$ or $X_{i} \in\{\mathrm{A}, \mathrm{B}\}$ for $V_{R}^{T}$ ).

$G_{*} \quad$ is the identifier of each of the nine types of grasps (Section IV-A).

Fig. 14 shows all the implemented fuzzy rules. The fuzzy operator of the antecedent is the AND computed with the minimum method, the consequent is computed using the minimal implication method, and when the same output variable appears in different rules the maximum aggregation method has been used [30].

The output of the FLC is a vector, $\alpha$, with 9 elements $\alpha_{*}$ representing the membership level of the actual configuration of user hand to each of the considered grasping types $G_{*}$, i.e.,

$$
\alpha=\left(\alpha_{T I e}, \alpha_{T I f}, \alpha_{T I M e}, \alpha_{\text {TIMf }}, \alpha_{\text {TIMRe }}, \alpha_{\text {TIMRf }}, \alpha_{\text {tripod }}, \alpha_{\text {sphere }}, \alpha_{\text {disk }}\right)
$$

Finally, $\alpha$ is processed to obtain an integer variable $G T$ that indicates the result of the grasp type identification, i.e. $G T \in\{0,1, \ldots, 9\}$ such that $G T=0$ means that the grasp was not identified as belonging to any of the nine considered 


\begin{tabular}{|c|c|c|c|c|c|c|c|l|}
\hline rule \# & $V_{R}^{T}$ & $V_{F}^{T}$ & $V_{F}^{I}$ & $V_{F}^{M}$ & $V_{F}^{R}$ & $V_{A}^{I M}$ & $V_{A}^{M R}$ & THEN \\
\hline 1 & $\mathrm{~B}$ & $\mathrm{~A}$ & $\mathrm{~A}$ & $\mathrm{C}$ & $\mathrm{C}$ & - & - & $G_{T I e}$ \\
2 & $\mathrm{~B}$ & $\mathrm{~B}$ & $\mathrm{~B}$ & $\mathrm{C}$ & $\mathrm{C}$ & - & - & $G_{T I f}$ \\
3 & $\mathrm{~B}$ & $\mathrm{~A}$ & $\mathrm{~A}$ & $\mathrm{~A}$ & $\mathrm{C}$ & $\mathrm{C}$ & - & $G_{T I M e}$ \\
4 & $\mathrm{~B}$ & $\mathrm{~B}$ & $\mathrm{~B}$ & $\mathrm{~B}$ & $\mathrm{C}$ & $\mathrm{B}$ & - & $G_{T I M f}$ \\
5 & $\mathrm{~B}$ & $\mathrm{~A}$ & $\mathrm{~A}$ & $\mathrm{~A}$ & $\mathrm{~A}$ & $\mathrm{C}$ & $\mathrm{C}$ & $G_{T I M R e}$ \\
6 & $\mathrm{~B}$ & $\mathrm{~B}$ & $\mathrm{~B}$ & $\mathrm{~B}$ & $\mathrm{~B}$ & $\mathrm{~B}$ & $\mathrm{~B}$ & $G_{T I M R f}$ \\
7 & $\mathrm{~B}$ & $\mathrm{~B}$ & $\mathrm{~B}$ & $\mathrm{~B}$ & $\mathrm{~B}$ & $\mathrm{C}$ & $\mathrm{C}$ & $G_{\text {TIMRf }}$ \\
8 & $\mathrm{~B}$ & $\mathrm{~A}$ & $\mathrm{~A}$ & $\mathrm{~A}$ & $\mathrm{C}$ & $\mathrm{A}$ & - & $G_{\text {tripod }}$ \\
9 & $\mathrm{~B}$ & $\mathrm{~A}$ & $\mathrm{~A}$ & $\mathrm{~A}$ & $\mathrm{C}$ & $\mathrm{B}$ & - & $G_{\text {tripod }}$ \\
10 & $\mathrm{~B}$ & $\mathrm{~B}$ & $\mathrm{~B}$ & $\mathrm{~B}$ & $\mathrm{~B}$ & $\mathrm{~A}$ & $\mathrm{~A}$ & $G_{\text {sphere }}$ \\
11 & $\mathrm{~B}$ & $\mathrm{~A}$ & $\mathrm{~A}$ & $\mathrm{~A}$ & $\mathrm{~A}$ & $\mathrm{~A}$ & $\mathrm{~A}$ & $G_{\text {disk }}$ \\
12 & $\mathrm{~B}$ & $\mathrm{~A}$ & $\mathrm{~A}$ & $\mathrm{~A}$ & $\mathrm{~A}$ & $\mathrm{~B}$ & $\mathrm{~B}$ & $G_{\text {disk }}$ \\
\hline
\end{tabular}

Fig. 14. Implemented fuzzy rules. There is one rule for each type of prismatic grasp (rules 1 to 6), with the exception of the $G_{\text {TIMRf }}$ that needs another rule (rule 7) to include larger abduction ranges. Regarding the circular grasps, due to the larger abduction ranges there are again 2 rules for Tripod grasps (rules 8 and 9) and 2 for Disk grasps (rules 11 and 12), while for the Sphere grasps just 1 rule was enough (rule 10).

types, and $G T=1, \ldots, 9$ indicates the identified type of grasp $G_{*}$ according to a predefined order.

\section{Mapping the identified type of grasp}

Once the hand pose of the operator has been recognized and represented with a value $G T \neq 0$, it is used together with the glove information (set of sensor values $S_{*}^{*}$ ) to compute a new grasp mapping of the user hand pose to the mechanical hand.

From the observation of the movements that a human performs to grasp an object with a precision grasp, it can be noted that, quite often, during the movement the distal and middle joints of the fingers do not change significantly, while the proximal joints do it (see an example in Fig. 15). Thus, the distances between the thumb tip and the other fingertips depend mainly on the proximal joints. Based on this observation the mechanical hand movement can be simplified significantly. In fact, from a practical point of view, for a number of grasp actions the fingers move only the proximal and abduction joints in a correlated way, and therefore the hand behaves like a device with only one dof. This consideration is used to simplify the commands to the mechanical hand based on the information obtained from the hand of the human operator.

For each type of grasp two vectors $L_{\min }$ and $L_{\max }$ of dimension $1 \times 13$ are determined, containing the joint values when the hand is totally closed and totally open, respectively (see Fig. 16 for some examples), i.e. the distance between the thumb tip and the remaining fingertips is minimal and maximal at the hand configuration defined, respectively, by $L_{\min }$ and $L_{\max }$. All the intermediate configurations of the human hand are reproduced on the robotic hand by linear interpolation between the configurations defined by these two limit vectors. This solution ensures collision free movements when a particular type of grasp is performed.

Algorithm 5 implements the pose mapping corresponding to $G_{\text {TIMRe }}$. It starts normalizing the values of the proximal sensor of each finger that takes part in the grasp (in this case the auxiliary variables I, M and R corresponding to the

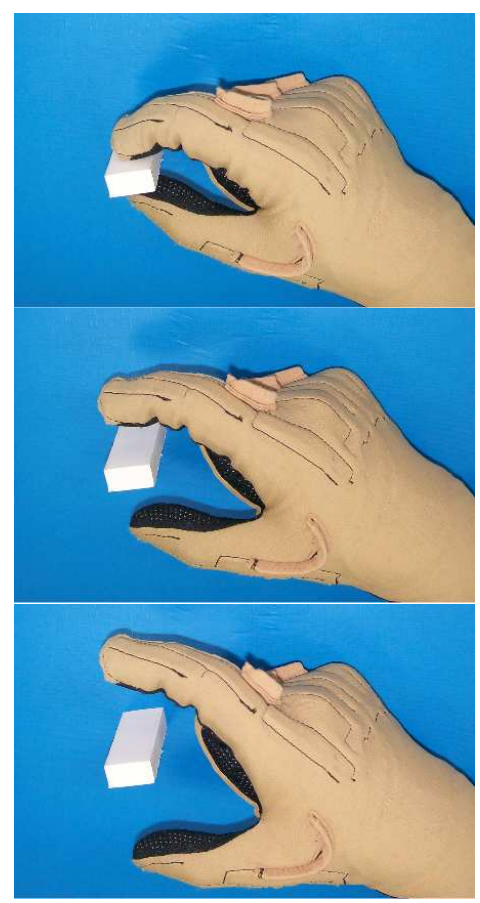

Fig. 15. Example of the finger movements doing a precision grasp.

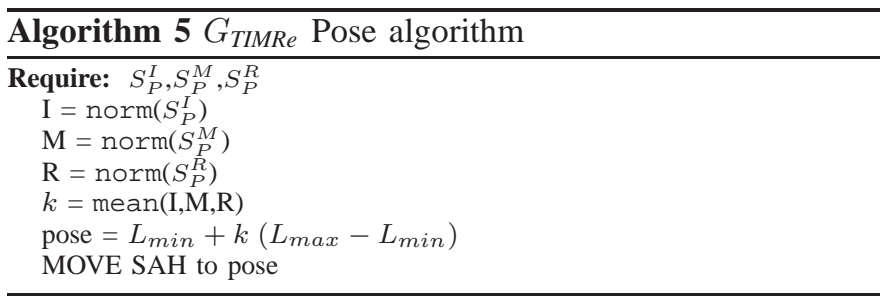

index, middle and ring fingers respectively); then, it computes the average $k$ of these values that is used to interpolate the position of the hand between the limits $L_{\min }$ and $L_{\max }$ of the corresponding type of grasp. Finally, the SAH hand is moved to the interpolated pose.

For the types of grasp that do not use the middle and ring finger (i.e. $G_{T T e}$ and $G_{T I f}$ ) or do not use just the ring finger (i.e. $G_{\text {TIMe }}, G_{\text {TIMf }}$ and $G_{\text {tripod }}$ ), Algorithm 5 is simply modified by removing the computation of the auxiliary variables $\mathrm{M}$ and $\mathrm{R}$, or just R, respectively, and removing them from the computation of the average $k$. It must be also reminded that the limits $L_{\min }$ and $L_{\max }$ are different for each type of grasp, and thus they must be properly selected in each case.

\section{HYBRID MAPPING}

The joint space mapping and the fuzzy-based pose mapping described in the previous two sections are now merged into a single hybrid mapping that acts as an assistance guide for teleoperated grasping tasks. Basically, the system monitors the information from the sensorized glove and computes the variable $G T$ that results from the identification process of the grasp type (Subsection IV-B), if $G T=0$ then a default mapping is executed (the joint space mapping described in Section III), and if $G T \neq 0$ then a particular predefined mapping is executed according to the value of $G T$ (the 

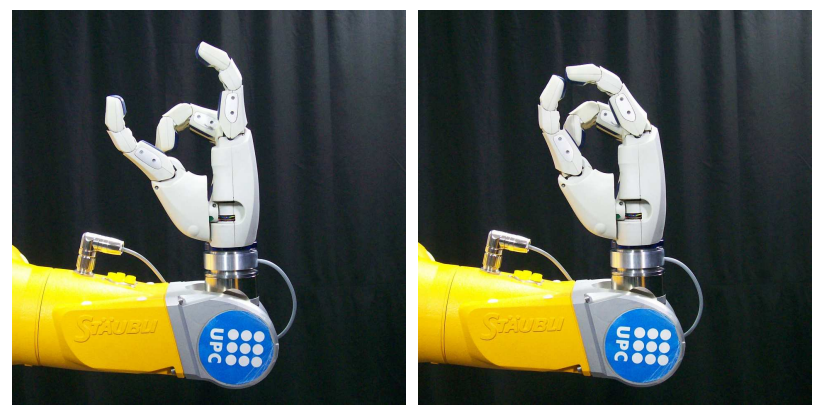

$G_{T I}$
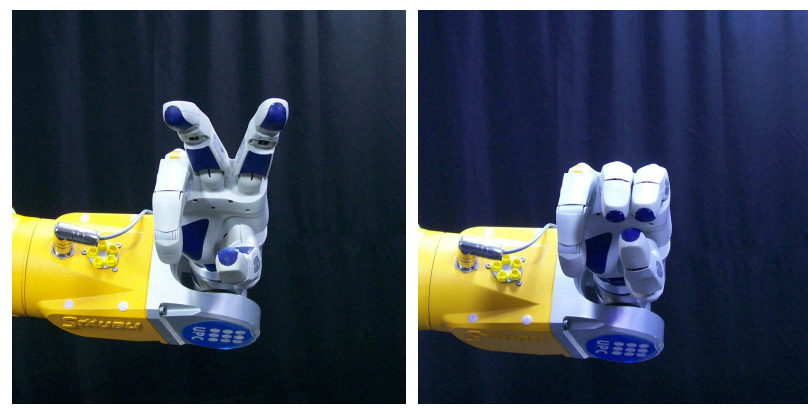

$G_{\text {tripod }}$
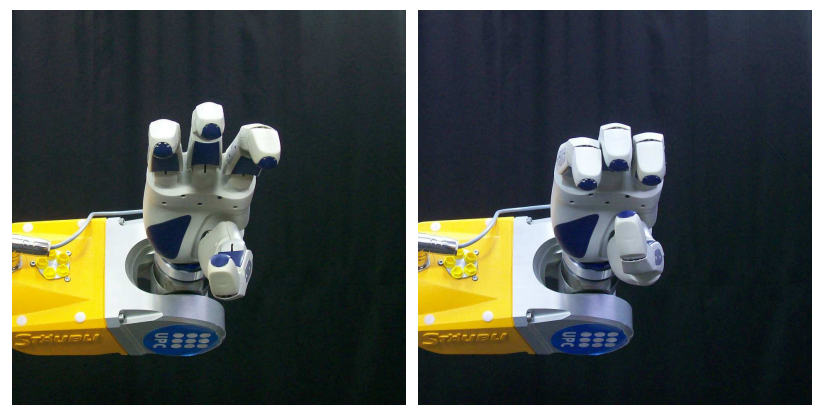

$G_{\text {sphere }}$

Fig. 16. Illustration of the fully open and fully closed configurations for different types of grasp.

fuzzy-based pose mapping described in Subsection IV-C). Fig. 17 illustrates how the hybrid mapping works based on the information from the sensorized glove and the variable $G T$.

\section{EXPERIMENTAL VALIDATION}

To perform the experimental validation, the setup described in Section II is controlled with two workstations:

- The local workstation receives the information of the dataglove (which is connected through the USB port) and executes the mapping, which is implemented in MatlabSimulink, obtaining the setpoints for the SAH hand.

- The remote workstation controls the SAH hand through the API functions using the setpoints received from the local workstation. The hand is mounted on the Stäubli TX-90 robot.

The communication between the workstations is done through Ethernet with a client-server architecture implemented in $\mathrm{C}++$, with the server running on the remote workstation and the client on the local one [31].

After setting up the system, different tests have been performed to fine-tune the algorithm parameters and to check the

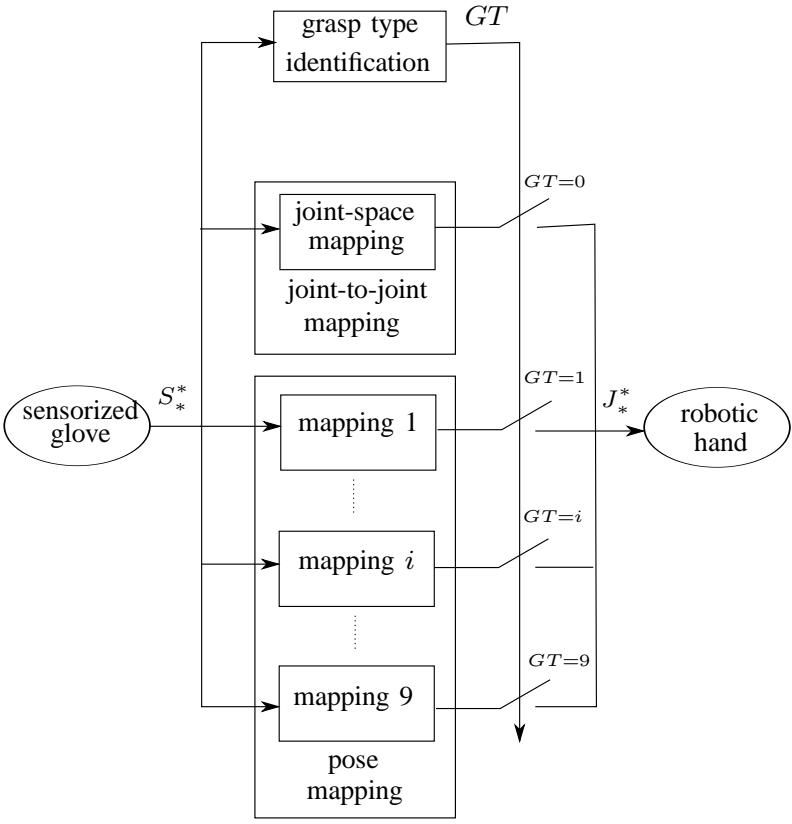

Fig. 17. Operating schema of the hybrid mapping, merging the joint space mapping and the fuzzy-based pose mapping.

\begin{tabular}{c|cccc}
$V_{F}^{*}$ & $V_{F}^{T}$ & $V_{F}^{I}$ & $V_{F}^{M}$ & $V_{F}^{R}$ \\
\hline$a_{1}$ & 0.7 & 0.3 & 0.25 & 0.15 \\
$a_{2}$ & 0.3 & 0.6 & 0.75 & 0.6 \\
$a_{3}$ & 0 & 1 & 0.3 & 1
\end{tabular}

TABLE I

VALUES OF $a_{1}, a_{2}$ AND $a_{3}$ USED FOR EACH FUNCTION $V_{F}^{*}$

level of correspondence reached between the human hand and the mechanical hand by the hybrid mapping. The values finally selected in the implementation were $\mu=\pi / 15$ (6 degrees) in Algorithm 2, and the values shown in Table I for the variables $a_{1}, a_{2}$ and $a_{3}$ of Eq. 4. The used input membership functions (IMF) are describe in the Appendix.

The transition between the different mappings may produce small discontinuities in the set points of the hand joints. This happens during free space movements and therefore was not a problem. Nevertheless controlling these transitions to obtain completely smooth movements of the hand could still improve the system performance. In the implemented approach, the pose mapping has priority over the joint-to-joint mapping. As a result, it is easy to command the hand to perform a grasp (use the pose mapping) but the reduction of dof implies a loss of dexterity and, if for some reason it is necessary, it is not intuitive to recover it (return to the joint-to-joint mapping). The only intuitive action for this is to open the hand significantly. This is a point for future work.

Fig. 18 illustrates the results of applying the joint space mapping, it can be seen that the correspondence between the finger configurations of the glove and of the $\mathrm{SAH}$ hand is satisfactory.

Fig. 19 illustrates the results of applying the fuzzy-based pose mapping for two types of grasps, a small prisms is grasped with $G_{T I e}$ and a medium size sphere is grasped with 
$G_{d i s k}$. Different grasp types have been tested with objects with different shapes and dimensions, leading to similar qualitative results that allow the mapping validation.

The experiments have been performed by different members of the Robotics Lab using only visual feedback, i.e. without any haptic device. The goal in the experiments was being able to do gestures with the mechanical hand and grasping objects to lift them, without any quantitative measure of grasp quality. The experiments show that, even with some background on robot manipulation, commanding grasping actions with a 13 dof mechanical hand requires specific training to become familiar with a system that has two non-identical kinematics (even being both anthropomorphic). The reduction from 13 $d o f$ to only one dof significantly helps the user in grasping actions because the operator only needs to focus the attention on the global position of the hand assuming that the fingers are closed to the desired pose. In any case, it must be note that any task could always be solved using only the joint-to-joint mapping.

\section{CONCLUSIONS AND DISCUSSION}

The paper has presented a new approach for the mapping of the movements of a human hand to a robotic anthropomorphic hand. The contributions of the work are the development of a joint space mapping (belonging to the joint-to-joint mapping type) and a fuzzy-based pose mapping (belonging to the pose mapping type) and the combination of them to produce a hybrid mapping that allows both intuitive free space movements (where the gesture is more important than the precise positions of the fingers) and grasp movements (where the precise positions of the fingers is more important than the gesture), always despite the kinematic differences between the human hand and the robotic hand.

The hybrid approach has been implemented and some illustrative execution examples are presented in the paper. The experiments have been done by members of robotics laboratory and therefore with knowledge and some training in robotics manipulation. The easy of use comments and the advantages of one mapping compared with another are based on the experiences of these persons, which agree with the expected results that justified the realization of the work. The system was not tested with people without any experience in order to obtain quantitative results on the dexterity reached with the different mappings.

Future work has two lines of activities. One is improvement of the individual mappings. Joint space mapping could be improved by using a non linear correspondence between the glove sensor values and the robotic hand joints. This would requires a complex experimental analysis of the desired relation between the hands to establish the improved correspondence. The fuzzy-based pose mapping could be improved using the synergies between human finger joints in order to determine in a simpler way more complex robotic hand movements. The second line of research is related to commutation between the mappings. In current implementation, pose mapping has priority over joint-to-joint mapping, but it would be interesting to be able to change the priority, either by an operator decision

\begin{tabular}{|c|c|c|c|c|}
\hline$V_{*}^{*}$ & IMF A & \multicolumn{2}{|c|}{ IMF B } & IMF C \\
\hline$V_{R}^{T}$ & 0.200 .30 & 0.150 .30 & - & - \\
\hline$V_{A}^{I M}$ & 0.500 .60 & 0.400 .60 & $\begin{array}{lll}0.75 & 0.95\end{array}$ & $\begin{array}{lll}0.70 & 0.80\end{array}$ \\
\hline$V_{A}^{M R}$ & 0.500 .60 & 0.400 .60 & 0.750 .95 & 0.700 .80 \\
\hline$V_{F}^{T}$ & 0.300 .40 & 0.300 .40 & 0.700 .80 & 0.700 .80 \\
\hline$V_{F}^{I}$ & 0.220 .35 & 0.220 .35 & 0.550 .65 & 0.550 .65 \\
\hline$V_{F}^{M}$ & 0.250 .35 & 0.250 .35 & $\begin{array}{lll}0.70 & 0.75\end{array}$ & $\begin{array}{lll}0.70 & 0.75\end{array}$ \\
\hline$V_{F}^{R}$ & 0.260 .35 & 0.260 .35 & 0.750 .83 & 0.750 .83 \\
\hline
\end{tabular}

Fig. 20. Values defining the rising and falling edges of the membership function A, B and C for each variable $V_{*}^{*}$ (see also Fig. 12). Function A has only two values that define its falling edge, function $\mathrm{C}$ has also two values defining its raising edge, and function $\mathrm{B}$ has two values defining the raising edge for $V_{R}^{T}$ and four values for the rest of the cases defining the raising edge and the falling edge respectively.

or, even better, automatically according to the task to be performed.

\section{APPENDIX: INPUT MEMBERSHIP FUNCTIONS}

The transitions from 1 to 0 of the input membership functions are defined by two values, $a$ and $b$, that represent the initial and final point of the transition; the input membership function IMF is defined as:

$$
\begin{cases}1 & \text { for } \quad x \leq a \\ 1-2((x-a) /(b-a))^{2} & \text { for } \quad a<x \leq(a+b) / 2 \\ 2((b-x) /(b-a))^{2} & \text { for } \quad(a+b) / 2<x \leq b \\ 0 & \text { for } x \geq b\end{cases}
$$

The transitions from 0 to 1 of the input membership functions are analogously defined. The actual values defining the edges of the membership functions are given in Fig. 20.

\section{REFERENCES}

[1] L. Basañez and R. Suárez, "Teleoperation," in Springer Handbook of Automation, S. Nof, Ed. Springer-Verlag, 2009, pp. 449-468.

[2] Y. Nam, K. Wohn, and H. Lee-Kwang, "Modeling and recognition of hand gesture using colored petri nets," IEEE Trans. on Systems, Man, and Cybernetics - Part A: Systems and Humans, vol. 29, no. 5, pp. 514-521, 1999.

[3] K. Abe, H. Saito, and S. Ozawa, "Virtual 3-D interface system via hand motion recognition from two cameras," IEEE Trans. on Systems, Man, and Cybernetics - Part A: Systems and Humans, vol. 32, no. 4, pp. 536-540, 2002.

[4] L. Gupta and S. Ma, "Gesture-based interaction and communication: Automated classification of hand gesture contours," IEEE Trans. on Systems, Man, and Cybernetics - Part C: Applications and Reviews, vol. 31, no. 1, pp. 114-120, 2001.

[5] J. P. Wachs, H. Stern, and Y. Edan, "Cluster labeling and parameter estimation for the automated setup of a hand-gesture recognition system," IEEE Trans. on Systems, Man, and Cybernetics - Part A: Systems and Humans, vol. 35, no. 6, pp. 932-944, 2005.

[6] I. Infantino, A. Chella, H. Dindo, and I. Macaluso, "Cognitive architecture for robotic hand posture learning," IEEE Trans. on Systems, Man, and Cybernetics - Part C: Applications and Reviews, vol. 35, no. 1, pp. 42-52, 2005.

[7] M.-C. Su, "A fuzzy rule-based approach to spatio-temporal hand gesture recognition," IEEE Trans. on Systems, Man, and Cybernetics - Part C: Applications and Reviews, vol. 30, no. 2, pp. 276-281, 2000.

[8] B. W. Miners, O. A. Basir, and M. S. Kamel, "Understanding hand gestures using approximate graph matching," IEEE Trans. on Systems, Man, and Cybernetics - Part A: Systems and Humans, vol. 35, no. 2, pp. 239-248, 2005.

[9] S. Cobos, M. Ferre, A. Sanchez-Uran, J. Ortego, and R. Aracil, "Human hand descriptions and gesture recognition for object manipulation," Computer Methods in Biomechanical and Biomedical Engineering, vol. 13 , no. 3 , pp. $307-317,2010$. 

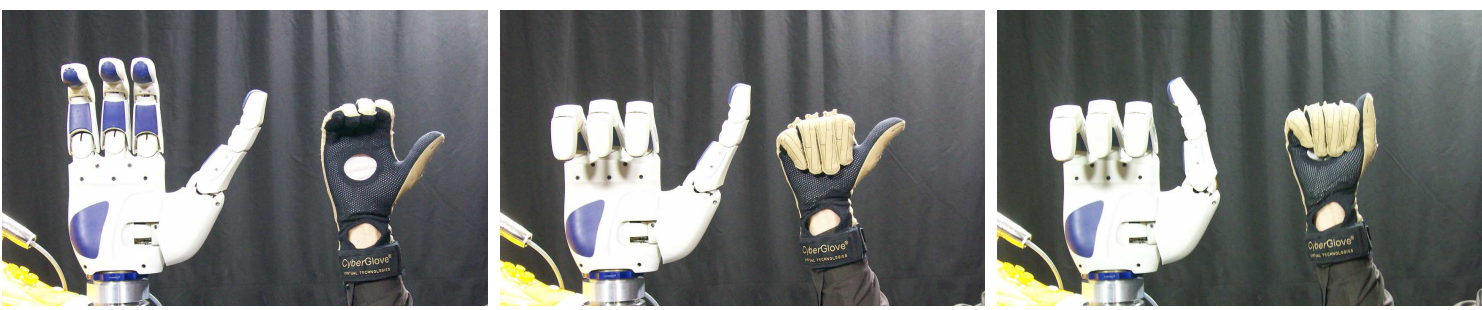

(a) Finger tests
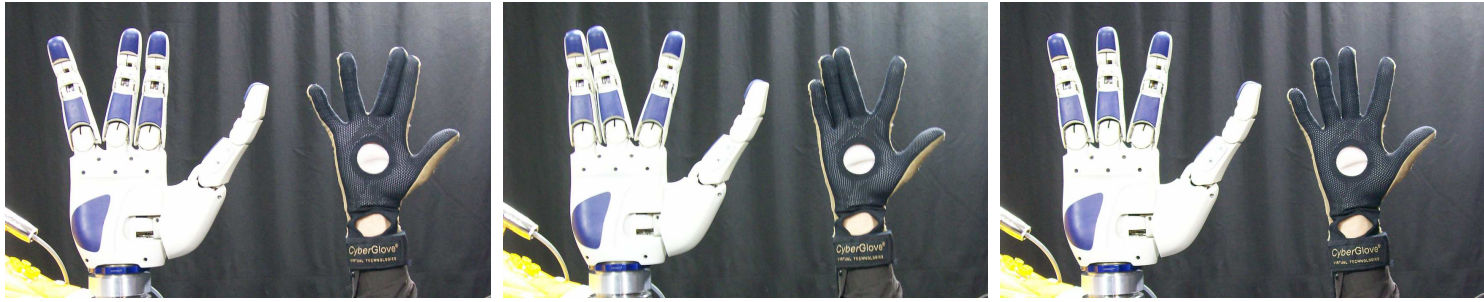

(b) Abduction test
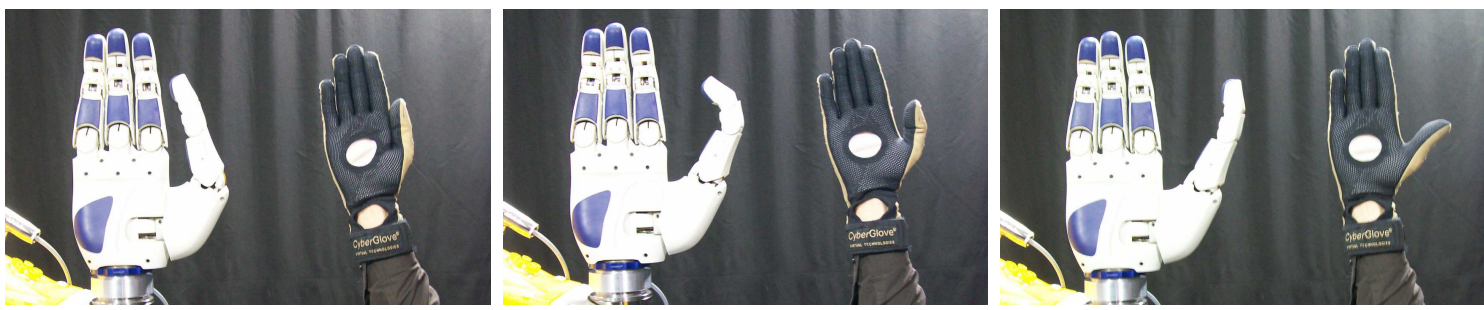

(c) Thumb test
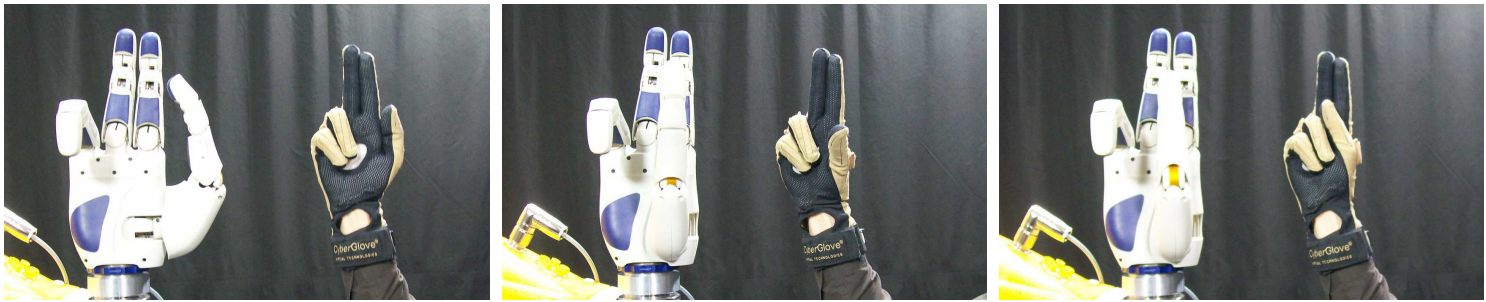

(d) Thumb-roll test

Fig. 18. Examples of the joint space mapping.

[10] J. Hong and X. Tan, "Calibrating a vpl dataglove for teleoperating the utah/mit hand," in Proceedings of IEEE Int. Conf. on Robotics and Automation, 1989, pp. 1752-1757.

[11] T. Wojtara and K. Nonami, "Hand posture detection by neural network and grasp mapping for a master slave hand system," in Proc. IEEE/RSJ Int. Conf. on Intelligent Robots and Systems, 2004, pp. 866-871.

[12] M. L. Tao Geng and M. Hülse, "Transferring human grasping synergies to a robot," Mechatronics, vol. 21, no. 1, pp. 272-284, 2011.

[13] J. Rosell, R. Suárez, C. Rosales, and A. Pérez, "Autonomous motion planning of a hand-arm robotic system based on captured human-like hand postures," Autonomous Robots, vol. 31, no. 1, pp. 87-102, 2011.

[14] H. Friedich, V. Grossmann, M. Ehrenmann, O. Rogalla, R. Zollner, and R. Dillman, "Towards cognitive elementary operators: Grasp classification using neural network classifiers," in Proceedings of IASTED Int. Conf. on Intelligent Systems and Control, 1999.

[15] J. Aleotti and S. Caselli, "Grasp recognition in virtual reality for robot pregrasp planning by demostration," in Proceedings of IEEE Int. Conf. on Robotics and Automation, 2006, pp. 2801-2806.

[16] J. Zhao, Z. Xie, L. Jiang, H. Cai, H. Liu, and G. Hirzinger, "EMG control for a five-fingered underactuated prosthetic hand based on wavelet transform and sample entropy," in Proc. IEEE/RSJ Int. Conf. on Intelligent Robots and Systems, 2006, pp. 3215-3220.

[17] T. Kondo, O. Amagi, and T. Nozawa, "Proposal of anticipatory pattern recognition for EMG prosthetic hand control," in Proc. IEEE Int. Conf. on Systems, Man and Cybernetics, 2008, pp. 897-902.

[18] T. Speeter, "Transforming human hand motion for telemanipulation," Presence, vol. 1, no. 1, pp. 63-78, 1992.

[19] R. Rohling, J. Hollerbach, and S. JAcobsen, "Optimized fingertip mapping: a general algorithm for robotic hand teleoperation," Presence, vol. 2, no. 3, pp. 203-220, 1993.

[20] A. Peer, S. Einenkel, and M. Buss, "Multi-fingered telemanipulation mapping of a human hand to a three finger gripper," in Proc. of the 17th IEEE Int. Symp. on Robot and Human Interactive Communication, 2008, pp. 465-470.

[21] L. Pao and T. H. Speeter, "Transformation of human hand positions for robotic hand control," in Proceedings of IEEE Int. Conf. on Robotics and Automation, 1989, pp. 1758-1763.

[22] J. Napier, "The prehensile movements of the human hand," Journal of Bone \& Joint Surgery, vol. 48B, no. 4, pp. 902-913, 1956.

[23] M. R. Cutkosky, "On grasp choice, grasp models, and the design of hands for manufacturing tasks," IEEE Trans. on Robotics and Automation, vol. 5, no. 3, pp. 269-279, 1989.

[24] L. A. Zadeh, "Fuzzy sets," Information and Control, vol. 8, no. 3, pp. 338-353, 1965.

[25] Schunk GmbH \& Co. KG, "Schunk anthropomorphic hand," http://www.schunk.com/, May 2006.

[26] J. Butterfass, M. Fischer, M. Grebenstein, S. Haidacher, and G. Hirzinger, "Design and experiences with DLR hand II," in Proc. of the World Automation Congress, vol. 15, 2004, pp. 105-110.

[27] J. Rosell, R. Suárez, C. Rosales, J. A. García, and A. Pérez, "Motion planning for high DOF anthropomorphic hands," in Proc. of the IEEE Int. Conf. on Robotics and Automation, 2009, pp. 4025-4030.

[28] E. Mamdani and S. Assilian, "An experiment in linguistic synthesis with a fuzzy logic controller," Int. Journal of Man-Machine Studies, vol. 7 , no. 1, pp. 1-13, 1975. 

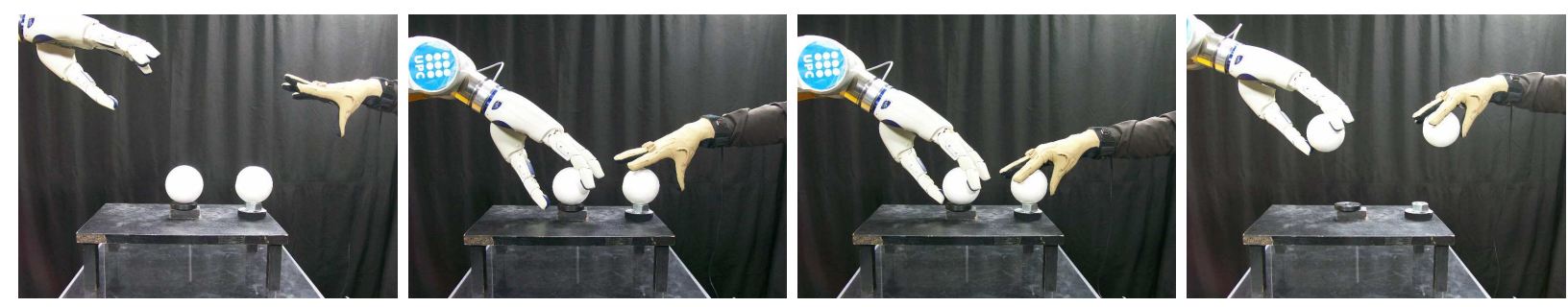

(a) Grasp type $G_{T I e}$
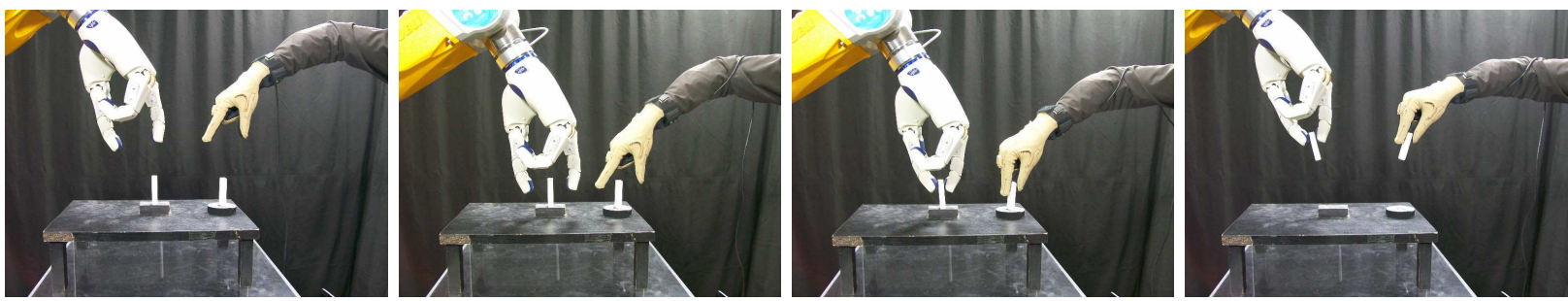

Fig. 19. Examples of the fuzzy-based pose mapping.

(b) Grasp type $G_{\text {disk }}$

[29] C. L. Taylor and R. J. Schwarz, "The anatomy and mechanics of the human hand," Artificial Limbs, vol. 2, no. 2, pp. 22-35, 1955.

[30] E. Mamdani, "Applications of fuzzy logic to approximate reasoning using linguistic synthesis," IEEE Trans. on Computers, vol. 26, no. 12, pp. 1182-1191, 1977.

[31] J. Fortín and R. Suárez, "General environment for human interaction with a robot hand-arm system and associate elements," in Proc. of the 15th IEEE Int. Conf. on Emerging Technologies and Factory Automation, 2010, pp. 34-39.

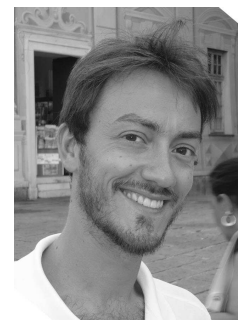

Luca Colasanto obtained the bachelor degree (with honors) and the master degree (with honors) in Control Systems Engineering from the Politecnico di Bari, Italy, in 2007 and 2010 respectively. During his master he was at the Institute of Industrial and Control Engineering (IOC) at the 'Universitat Politècnica de Catalunya' (UPC), Barcelona, Spain. $\mathrm{He}$ is currently a Ph.D. student at the Fondazione Istituto Italiano di Tecnologia, in Genova, Italy. His current research is in humanoid robots, dynamic walking control and soft robotics.

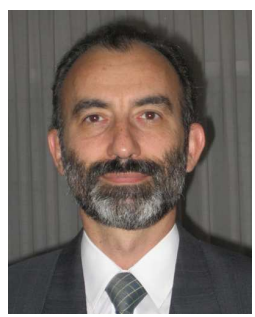

Raúl Suárez (M’96) received the electronic engineer degree (with honors) from the National University of San Juan, Argentina, in 1984, and the $\mathrm{Ph} . D$. degree (cum laude) from the "Universitat Politècnica de Catalunya' (UPC), Barcelona, Spain, in 1993. He is Research Supervisor at the Institute of Industrial and Control Engineering (IOC), UPC, where he has been responsible for the research line Process Control (1998-2003), Assistant Director (2003-2009) and, at present, he is the Director (since 2009) and the Coordinator of the Doctoral Programs Advanced Automation and Robotics (since 1995), and Automatic Control, Robotics and Computer Vision (since 2006). His main research areas include grasping and manipulation, mechanical hands, fixturing, assembly, task planning, telemanipulation and manufacturing automation.

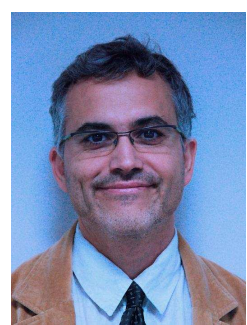

Jan Rosell received the BS degree in Telecomunication Engineering and the Ph.D. degree in Advanced Automation and Robotics from the 'Universitat Politècnica de Catalunya' (UPC), Barcelona, Spain, in 1989 and 1998, respectively. He joined the Institute of Industrial and Control Engineering (IOC) in 1992 where he has developed research activities in robotics. He has been involved in teaching activities in Automatic Control and Robotics as Assistant Professor since 1996 and as Associate Professor since 2001. His current technical areas include robot motion planning, haptics, robotic assembly and manufacturing automation. 